\title{
8 Stasis, observation, and facts
}

A very long time ago I descended into the lower school in a private school when I was principal to substitute for a seventh grade teacher. I had in previous years taught high school science in the upper school. Needing to improvise as I had no lesson plans I thought it best to start a general discussion about science with the class. To stir them up a little I raised the question about the differences between facts and theories. I almost started a class rebellion among those bright little charges when I said to provoke them that it was not a fact that the moon circled the earth.

Every last one of them insisted that I was wrong. It was a statement that countered everything that they had come to learn and believe from the popular astronomy that saturated their everyday pop culture with its Copernican view that the sun was the center of the solar system and that moons circled planets. All statements about these matters were considered by them to be statements of fact. And how, oh how now do so many people think as those young students thought that science is all about facts, and that teachers create tests to measure the extent of student knowledge of a pile of rote learned scientific facts?

How much is the public bamboozled into thinking that some seemingly warranted scientifically conjectures, theories, many scientific concepts are simply facts. Our accepted scientific theories are considered to be simply facts. But how many of these theories turn out to be useful but glorified fictions? Ironically, what I was saying to those students sounded very much like that famous papal contention between the pope and Galileo where the papal view was that it was not a fact but a theory that the sun was the center of the universe. Obviously now the pope at the time was right as no astronomer now that I am aware of considers the sun as standing still in the center of the universe.

The sun moves around the galaxy of the Milky Way. And the Milky Way is going I know not where and in what directions, for directions seem to make no sense when things are moving without any fixed point of reference or point of stability to know exactly in which they are going. But where then in all this commotion going on in the heavens if there is no center? From our everyday perspective now we are now feeling comfortably sitting or standing on a fixed point on the earth within the solar system with the stars and galaxies all about us. At least that perspective gives us our useful everyday sense of stability.

To get those students to start thinking I tried to convince those young boys, as it was the Harvard School for Boys, that when we observe facts we see them happening. They are to be found in our witnessing of events, and that they are found in our observations of things from the position where we stand. They are about events and objects in certain perspectives. They are about the things that we see happening. They are things that get done. But to the contrary what we see as facts in our view of the heavens as I pointed out to them is not the moon circling the earth. The statement that the moon circles the earth is a theory used to explain certain facts, but that point of 
view of seeing the path of the moon is not actually an observable fact. We do not see exactly what is happening, but can only surmise through hypotheses or conjectures what is possibly happening.

This little incident with students happened many years before the astronauts first landed on the moon. It is interesting to examine what the astronauts saw when they landed on the moon. What the astronauts had to say of what moved in the heavens I have found in my reading has been far too sparse, and what they had to say was not based upon accurately measured descriptions. From the moon the earth, unlike our moon that moves across the sky, displays its rotation while the moon that we see does not rotate as we see it moving across the earth sky. We do not see the moon visibly rotating. We see mostly just one side.

Observation of the sun and the earth on the moon is much the same sort of thing as observations of the sun and the moon on the earth. But they vary depending upon what one sees from different stand points and different places. Just as what we observe of the movements of the moon across the sky at various times and places depends on our geographical locations, we should then expect the same sort of variations of what goes on in the sky to be seen differently from various locations on the lunar landscape.

Note also that what the moon astronauts were also seeing is what astronomers do when they are observing the units of measurements on the instruments that they were using in measuring what they are observing on the moon. There is a radical difference between seeing the qualitative facts and the facts of the measurement that we see recorded on our measuring instruments. Traditionally on earth we used the astrolabe and sextant and a chronometer to make our measurements.

However, in discussing with my students about what we see of the moon in the sky I avoided all talk about making any measurements about movements of the moon. I was simply playing games with them semantically and with the paradoxes of what it means to 'go around' (Yoos, 2009). To illustrate ambiguity I often use that famous paradox discussed by William James about a squirrel going around a tree. Note that what I was doing to the young charges, besides making them think, was proposing to them new ways of talking about what we begin to understand when we propose different technically defined definitions about what are 'facts' and what are 'theories'. I had been in my own teaching of high school science using these very same defined distinctions to help clarify concepts in classes. I did that to make my high school students fully aware of the confusions we get into when we think of certain theories as facts. There arises a great disconnect in ordinary English when we want to say that scientific laws or principles are the true facts about the world.

What I illustrated to my high school students is that in one way of talking, we establish facts in one way directly by the senses and that we use them to warrant our theories quite differently. Theories I indicated are more like conjectures that need not be exactly accurate or exactly true. A conjecture or an assumption in one sense of either of these two terms is simply a hypothesis about which we can never be absolutely certain about, especially when there are ambiguities and vagueness in the 
statements of theory that make them confusing about the accuracy and truth of what is being conjectured about.

But then to confirm my view to the elementary students that the moon does not in fact circle the earth I attempted to show them that the earth and the moon's orbit with reference to the sun was not exactly a circle, and that earth and the moon were constantly chasing each other in their orbits and that those orbits were not simply and unambiguously a circle, but that both the earth and the moon were said to be rotating together travelling along together in separate ellipses around the sun, both seeming going around the sun whirling, paired together in a constant rotation in a state of equilibrium in the same direction.

And then I tried to show them from that one point of view that the elliptical orbits of the earth and the moon together were undulating curves just moving up and down along interacting changing paths going after and chasing each other around the sun. And then I explained that their movements were attracting each other by gravitation, and that they were actually chasing each other as they moved away from each other, each swerving in orbits that escaped centripetally the path of the other. Moving along the earth and moon in their solar elliptical orbits they were not simply circling but chasing each other as they moved in their orbits around the sun.

What I pointed out, to make my point, was the ambiguity of what we were considering to be a fact, that a fact in one sense of the term is what we actually observe to be a fact, what happens, and when we watch the moon we observe certain facts about its movement. What we see as a fact is that the moon circles the earth from our perspective where we are standing still and that the moon moves over us from the eastern to the western horizon. And that same so called fact was equally thought to be true in the old Ptolemaic theory in Greek astronomy. The moon circled the earth across the sky very much like the sun. And then I showed them that what was being said about the sun and moon circling the earth depended on what we see from the very place where we stand. Movement as we perceive it is always relative to a stationary perspective. It is all about being static or being in stasis.

Then I tried to explain to them that an explanation or theory depends upon our interpretations of the facts, but many of us think that those statements that logically follow from our theories thought to be true have to be facts. But theories are not facts but conjectures about what happens from certain points of view. What we would see if we were located somewhere else in space where we could observe what follows from our logical interpretations of our theories would then be observed as facts. I suggested to them that theories are about supposed or conjectured facts, that they are not statements about the facts themselves. I was thus proposing to them a different way of thinking that cleared away some of the confusions about the difference between what it is to be considered to be a fact and a theory in ordinary ways of speaking. Theories in this redefined sense are conjectures about what is thought to be true. They are about things we do not directly observe, and our thinking that they are true ultimately depends upon our interpretation of our observations that the theory tries to explain. 
Given time if I had been their teacher I could have shown them that the view of the solar system from the view point of Copernican astronomy, which if true, presupposes that the solar system is supposedly in stable equilibrium, either in stasis or standing still. But again to repeat if the sun is moving around the center of the Milky Way, and the Milky Way is moving with respect of the galaxies, where is there any place for an observation of facts of the sort where we could see what is moving and with reference to what? Or to put it in different words, if we want a stable perspective of the universe where would we need to stand? Note that the theory of an expanding universe is a theory that takes the perspective of the earth as the stable center of these measureable expansions that are implied by the red shift in the light spectrums coming from the stars. Note that to say that everything is expanding away from everything is not an observable fact, but a theory that seems to be a theory thought true by astronomical cosmologists in their interpreting the differences in red shifts measurements in the light spectrum that appears to be coming from galaxies.

In discussing astronomical questions about facts and theories we seem to be left only with our quest for stability. We as human beings are seeking a place to stand that defines our own sense of stability as a reference point for making all the other measurable orientations between different things that seem coherently related. Man in that sense is the measure of all things. What seems required is a standpoint for measurement that provides an accurate description of the ultimate changes that seems to be occurring. But that leads to all sorts of messy conceptions of space and time.

If my contention is right, it is not the complexity out there in the world that confuses us, it is the messiness that we have in theories or models that we use to describe what seems to be a random sort of messiness in the universe with no actual boundaries and that has no order. But what actually is messy is the messiness of the mathematical models that we are using to describe the messiness of the facts that we see. What leads to messy contentions about which way the moon moves comes from the inconsistencies found in our formal systems that we use to model what seem us to be correct explanations or theories.

When we talk about the formal inconsistencies of our scientific theories, especially when we try to find a model that contains infinite changing directions and infinite extensions, nothing seems bounded or referenced to a standpoint in them whereby we can build a model or theory that encompasses everything, that is, 'a theory of everything'. In other words, to have a theory that explains 'everything' requires an absolute consideration of all the facts and that those facts are what they purport to be such as the way we observe the moon circling above through the skies that only circles around us from the factual standpoint we have in looking up at it as it traverses the sky.

Note that in looking up in tilting my head it is difficult to maintain stable equilibrium. But note too what happens when I observe things when I am having vertigo. What are the facts that I observe directly when one's horizon is spinning? 
What we observe as facts is changing in vertigo from what I observe when I am standing tall and visually stable. Changes in equilibriums, changes of the balances of things around something static, is one way then to measure change.

But the changes we see are also ways that we have of determining our references to things. If I lose my balance I try to stabilize myself by trying to stand up. In starting to fall down I have lost my equilibrium. In its physical descriptions my body in falling down is changing from a stable equilibrium of standing tall into a dynamic equilibrium of being a falling object that no longer is in equilibrium with the gravitational pull of the earth. To regain my feet I either push myself back up, or in my old age someone needs to help me to stand back up again. And if I have a walker I might pull myself up to get back up on my feet. The key to regaining stability or regaining a stable dynamic equilibrium is some sort of feedback to regain my equilibrium or stability. You choose the wor1d to describe my stability. Stasis is a fancy word for it. Note that observation starts from what we think of as a static or in a set position.

It is obvious in studying mechanics in physics that our models of statics and dynamics interrelate when something is moving. In one way in physics we can study statics as a discipline separately, and in another way we can study dynamics separately. But something moving also can be in stasis or in a stable equilibrium. Feedback is the way we have of maintaining stable equilibrium and of preventing change, or it is the way we have of re-establishing and putting something again back into a stable equilibrium as in dynamics.

In politics the stable equilibrium of economic and legal systems is a view often labeled 'conservatism'. It is a view that in standing tall we never need to move from where we are. We are set in our ways about who we are. What is striking about life are the feed backs that are controlling and monitoring, and that are structured by sensory devices that maintain the equilibriums especially within the cell walls of living things. These walls or membranes allow the passage of what is needed from the outside to bring it into the inside to maintain the equilibriums existing within the enclosures or within the membranes that protect the organisms.

But from another point of view, from the view of those who devise devices to maintain types of mechanical equilibriums, we see that these mechanical models are varied and differ in many ways from the models that we make of those homeostatic creatures that are said to be alive. Mechanical devices have been produced by artifice, by human ingenuity. They have been devised by an intelligence that simplifies a variety of tasks that maintain different material states of equilibrium. Key then to this sort of designing and engineering of these mechanical types of equilibriums are the construction of technical devices that significantly differ in structure and form from what is monitoring and is going on within those cells that are governing what we think of as life.

It is these models and devices that we think of as machines that are at the heart of science and technology that manages and maintains these mechanisms. For example, we use mechanical electrical devices to create our external environment. They help us 
create environments within our buildings, or they manage and maintain our flows of transportation or our distribution of electricity. They possess external monitors that regulate feedback to maintain stasis in our external environments. All such stabilizing devices are based upon devices designed by human engineering. They are artifices. They all have feedback systems that are engineered creatively. But to what degree are the forms of life monitored within and not imposed and controlled from the artificers from on the outside?

My first awareness of feedback and equilibrium dawned on me at the age of nine. We lived by the railroad tracks watching the steam engines go by one after the other. My uncles and my dad with whom I lived at the time had all worked around the round houses serving two different railroads around Centralia, Illinois. It was a railroad town. But at that time I had not the good sense to ask them how those steam engines worked. Still I was curious about why all that huffing and puffing of steam that made those drive wheels turn that were pulling all those long and heavy freights and all those fast passenger trains that were flying by night and day on the Illinois Central. I watched those large cylinders connected to those drive shafts. And suddenly to my amazement I noted how that long shaft reversed itself and moved in the opposite direction to redirect the steam in the opposite direction to make the pistons reciprocate back and forth. It was my first sense of feedback and about what a governor is. It governs. But the governing was external to the engine. It regulated steam flow. It was an artificial mechanical invention.

Suddenly it occurred to me that I understood the function of what is now called a camshaft that reverses the direction of the exhaust and the steam, thus resulting in the huffing and puffing noise that was coming from the exhaust of those steam engines. In my youthful imagination I had found to my surprise that I had figured out how to design a steam engine. In my imagination I came to understand an example of a simple device used for reciprocating feedback that kept the train in moving in dynamic equilibrium. And if the engineer wanted more speed, he would release more steam into the cylinders with his throttle and the drive shaft fixed to the wheel would drive those wheels faster as the engine accelerated forward. And to maintain that dynamic equilibrium there was the governor. A spinning set of balls whirling in a circle that regulated the amount of steam that was flowing into those cylinders. It was a mechanical accelerator that allowed the train to speed up and then with the throttle adjusted to hold those rotations at a constant pace, thus maintaining and regulating the equilibrium of the train at a constant velocity.

And later in life before World War II in the dirtiest job I have ever had, I discovered all those intricate soot covered mechanisms that were used to regulate all the various steam engine drivers that regulated the steam pressures, that regulated the air valves on the brakes, and kept that steam engine in various states of equilibrium as it maintained its speed and direction in dynamic equilibrium running down the rail road tracks. And still later in the war I found a much cleaner kind of equilibrium using comparable feedback technology in the air planes in which I flew. Those old 
bombers were complicated combinations of feedback and backup systems. There I found all those devices that maintained equilibrium that when they were thrown out of equilibrium had to have all those various forms of feedback systems to restore again the flight of those planes to an original stable state of directional equilibrium.

Let me just list of few of those devices: there were Wheatstone bridges and gyros in the autopilots that stabilized the equilibrium of the flight control systems, there were gyros and magnetometers in the compass system, there were oscillators in the radio transmitters and their receivers in our communications systems, and there were oscillators in our radar systems and in our Loran. There were camshafts, carburetors, and distributers regulating different feedback devices in monitoring the engine and prop speeds. And we had pressurized cabins in B-29's that had thermostats and pressure stats that maintained the temperature and pressures in the cabins. And there were automatic feedback systems in the gunnery in the central fire controlled systems. And the optical bombsights were just one complicated set of electrical motors that automated the equilibrium of the bomb sighting that was in turn integrated with the bomb release systems with their timing circuits. Such was my early practical education I had in equilibrium models that explained what was going around me in those monstrous bomb truck carrying vehicles. They were human inventions, artifices that were not a product of biological evolution, but they were designed to kill thousands of people in the name of peace and the hope of a return to normalcy in our society, that is, to return to some sort of stable peaceful equilibrium that never really happened.

The intelligence and ingenuity of designing and testing all those mechanical systems were developed for warfare by the study of prototype models that were a product of the study of military testing and proving grounds. Some of it worked better than expected. But at the time the radar aiming devices were something only practicably applicable. They were designed not for precision bombing as the radar antennae had no vertical stability, and the radar ground returns had no precise definition of the targets. The radar returns were only shifting unstable and large globs of light that gave no accuracy. What were always at issue were their reliability and the overcoming of the imprecision with which these devices regulated the equilibrium needed for the force vectors behind and under the plane to go to where it was aimed and to do what it was supposed to do, and when it got there to dump their fire and demolition bombs on open cities to burn and bomb the hell out of homes and kill helpless people.

In many ways machines act in many ways in the same ways as biological organisms and that raises, as mentioned, the issue discussed whether or not these patterns of these biological functions were as law like just as physicists thought about the motions and the feedbacks of our mechanical mechanisms. Is there something inherently determinate and law like in the nature of beasts just like that which seems to govern machines? Or is it that these patterns used in our machines are just useful in the way as they help us organize our actions in the ways we have of designing 
machines, testing them, and in using them? In other words are our equilibrium theories about machines are only comparable but they are not the same as our models of human action based upon the functions of human bodies? But nevertheless we do create machines to simplify and help us order and govern our human actions in dealing with the various problems that we have to solve in getting things done.

It has been my contention that instead of thinking of models as representations of things or law like structures in the state of nature as equally the same as the processes of human life, we should look at models first as guides and prototypes by which we exercise command and control in dealing with the problems of life. And it is worth noting that, as we devise these models of human life processes to understand them, that these models in turn become more and more complicated. And using much more complicated models in order to understand life we need in turn to simplify our ways of understanding them so as to discover how we can best use them in ways that make our uses of them less complicated in explaining life functions.

When our schemes and models are too complicated as seems to be the case with life processes for any direct and holistic understanding of them, we need to thread our way through their complexities using the logic and the rules of the ways we have of constructing these models to see how we can in a much simpler fashion interpret our schematic models of life. Especially we need to see whether or not we can interpret them in such a way so as to apply them in economic and efficient ways of making life functions much healthier in living out of our lives.

But in the quest for such simplifications, the problem with using models for both mechanical machines and living processes is that they can be over simplistic, and when over simplified they have only limited applications. On the other hand, as these models become less simplistic they require modes of interpretation that are more and more complicated. And as we try to explain further their complications more and more complications are added to them again and again. When that happens we need again to see these complications in these increasingly complex frames again in simpler terms to better understand them.

To illustrate the problems of thinking about complexity, chess is an interesting model of how we need in overcoming the constraints of memory and our use of chessclock time to deal with these problem solving issues. Note that chess players do not simply calculate on the basis of the rules that generate the possible configurations of the game and then test them by their calculations using the formation and transformational rules of chess. That task would not be humanly possible within the limitations of human attention and the constraints on human short and long term memory. Even a computer does not have power to make such complicated calculations by simply using the rules of chess (Simon, 1979: 363-403).

Rather the skillful chess player simplifies his conception of play by learning strategies for beginning moves that are simpler and easier to define, and then after the game progresses they learn new strategies to use in the middle game where complications cannot be simply understood except by knowing which different 
configurations lead to which variations and the complications that develop out of original opening lines of play. Original lines of play hopefully are thought to lead to a set of favorable positions in the end game. It is in the middle of the game that mistakes are usually made as the positions are too complicated to be solved by any set of algorithmic strategic rules. It is at this point of the game that improvisation and difficult calculations have to be made to arrive at desired positions for an end game.

When a game of chess begins to deviate from known opening theories in chess, the players are said to be 'out of book'. In some opening lines the moves considered best for both sides have been worked out for ten to fifteen moves or more. Some analysis goes to thirty or thirty-five moves, as in the classical King's Indian Defense and the variations of the Sicilian Defense. And it is the end game that is aimed at in their studying and exploring the various lines of play that develops in the complexity from the original lines of play. In chess the complications of the endgame are much easier to master as the complexity has been reduced. And since chess must be constrained not only by the structure and pattern of the board, but chess games are constrained when playing in competition by the rules defining the time constraints that alter the possibilities of different strategies in using chess-clock time efficiently to do different calculations.

The need for simplicity in chess strategy is a result of the chess player's limits of memory and the limits of their personal abilities to calculate. Chess as a model illustrates well how there is a need to simplify in order to understand complexity and to predict results. Chess like all complex models needs simplification of our ways of dealing with complexity that again and again require more and more complicated models to deal with the complexity that we do not understand in using the beginning models of lines of play that we have used that are leading us into difficulties.

But the problem then of using simple models is that they mislead us eventually in how we interpret and misunderstand them. In my youth my concept of a bridge was a straight mantel across two pillars. But when I with other kids tried to build a bridge out of oil well cables, we made the mistake of tightening them in a straight line up high between two huge trees. Our bridge that we constructed and built with stretched tight straight line cables between two trees used as our static pillars was a total disaster. What happened is that we were actually using a model of a long or cross bow. We found to our despair that when the clamps that we used to tighten the cables to the opposite trees in a straight line surprisingly slipped, and the kids working on our cable bridge shot up like arrows into the air. We did not recognize that the model we were using was not one for building a suspension bridge, but it was that of a bow that stretches and releases its arrows from a taught string. Using a wrong model we created a disaster. What we believed as the proper way to build a bridge was false. We found knowledge and truth in our mistake.

And that has been a pattern that has often occurred in failures in the history of science. The model of heat as a fluid with negative weight failed to predict. The model of electricity as a positive charge fluid failed to predict. The model of Newton's 
mechanics did not predict the behavior of nuclear particles. And the model of light as corpuscles failed to predict interference effects of light. But despite these deficiencies in these models, though they are now discounted as theories, they still have turned out to have practical usefulness in certain limited contexts as many of these old models are still used in many technical applications.

What we know now as a result is that models though faulty are still useful in doing certain sorts of things, but they were not useful in telling us how to do certain other things. We know they have false applications. We know that many of their implications are false. That they are false is demonstrably so, and what they are false about has definitely become knowledge. When we work with testable hypotheses about empirical generalizations that we make from observations, we attempt to weigh the evidence that seems to support such generalizations. But that awareness of repetitive events never allows us to prove anything with certainty. 